Communications in Physics, Vol. 24, No.3S2 (2014), pp. 18-22

DOI:10.15625/0868-3166/24/3S2/5048

\title{
CONDUCTIVITY IN HALF-FILLED IONIC HUBBARD MODEL
}

\author{
NGUYEN DANH TUNG AND HOANG ANH TUAN \\ Institute of Physics, Vietnam Academy of Science and Technology \\ E-mail: hatuan@iop.vast.ac.vn
}

Received 20 June 2014

Accepted for publication 20 August 2014

\begin{abstract}
We calculate the temperature dependent conductivity in the half-filled ionic Hubbard model with an on-site Coulomb repulsion $U$ and an ionic energy $\Delta$ by mean of the coherent potential approximation. It is shown that for intermediate and large $\Delta$ the largest conductivity occurs near the special value $U=2 \Delta$ at all temperatures $T$, for a fixed $\Delta$ the region of finite conductivity $\left[U_{c 1}, U_{c 2}\right]$ expands and its maximum decreases with increasing $T$. Our results are in good agreement with those derived from the determinant quantum Monte Carlo simulation.
\end{abstract}

Keywords: static conductivity, ionic Hubbard model, coherent potential approximation.

\section{INTRODUCTION}

It is well established that an on-site Coulomb interaction between the valence electrons with opposite spins can lead to their localization in the lattice sites and can drive a transition to a chargegapped Mott insulator (MI). On the other hand, the imposition of an external periodic potential can drive a band insulator (BI) with one sub-band fully filled and the other one empty. The ionic Hubbard model (IHM) includes an on-site Coulomb repulsion and a staggered potential and is therefore well suited to study transitions between metallic and different insulating phases $[1,2]$.

Recently, the IHM in high dimensions has attracted much interest and it has been widely studied by a variety of techniques including Hartree-Fock theory, slave boson approach, determinant quantum Monte Carlo (DQMC) simulation, and dynamical mean field theory (DMFT) [3-9]. However, the precise conclusions about the phase diagram are still subject to some debate. In a previous paper [10] we have applied the coherent potential approximation (CPA) to study electronic phase transitions in the half-filled IHM in dimensions $D \geq 2$ at zero temperature. We found that MI and BI phases are sandwiched by a metallic one. In order to further analyze these phases, in this paper we will calculate the dc conductivity at finite temperature and consider its behavior in the model.

(C)2014 Vietnam Academy of Science and Technology 


\section{MODEL AND FORMALISM}

B)

We consider the following Hamiltonian for the IHM on a bipartite lattice (sublattices A and

$$
H=-t \sum_{<i j>\sigma}\left[c_{i \sigma}^{+} c_{j \sigma}+\text { H.c. }\right]+U \sum_{i} n_{i \uparrow} n_{i \downarrow}+\varepsilon_{A} \sum_{i \in A} n_{i}+\varepsilon_{B} \sum_{i \in B} n_{i}-\mu \sum_{i} n_{i},
$$

where $c_{i \sigma}\left(c_{i \sigma}^{+}\right)$annihilates (creates) an electron with spin $\sigma$ at site $i, n_{i \sigma}=c_{i \sigma}^{+} c_{i \sigma}$ and $n_{i}=n_{i \uparrow}+n_{i \downarrow}$. $U$ is the on-site Coulomb repulsion, $t$ is the nearest neighbor hopping parameter, $\varepsilon_{A}=\Delta$ and $\varepsilon_{B}=-\Delta$ are the ionic energies. The chemical potential is chosen so that the average occupancy is 1 (half-filling).

In order to investigate the Hamiltonian (1), we firstly consider an alloy problem which is expressed by the following Hamiltonian:

$$
H=\sum_{i \in A, \sigma} E_{A \sigma} n_{i \sigma}+\sum_{i \in B, \sigma} E_{B \sigma} n_{i \sigma}-t \sum_{i \in A, j \in B, \sigma}\left[c_{i \sigma}^{+} c_{j \sigma}+\text { H.c. }\right],
$$

where

$$
E_{\alpha, \sigma}= \begin{cases}\varepsilon_{\alpha}-U / 2 & \text { with probability } 1-n_{\alpha,-\sigma}, \\ \varepsilon_{\alpha}+U / 2 & \text { with probability } n_{\alpha,-\sigma},\end{cases}
$$

here $\alpha=A, B$ and $n_{\alpha, \sigma}$ is the average occupation with spin $\sigma$ in the $\alpha$-sublattice. As in [10] we focus ${ }^{1}$ on the paramagnetic case, for which: $n_{\alpha \uparrow}=n_{\alpha \downarrow}=n_{\alpha} / 2$. The Green function corresponding to the Hamiltonian (2) has to be averaged over all possible configuration of the random potential which can be considered to be due to alloy constituents. The averaging cannot be performed exactly. As a second approximation we apply the CPA to the alloy problem. The CPA demands that the scattering matrix should vanish on average. This yields an expression for $\Sigma_{\alpha}(\omega)$ of the form

$$
\Sigma_{\alpha}=\bar{E}_{\alpha}-\left(\varepsilon_{\alpha}-U / 2-\Sigma_{\alpha}\right) G_{\alpha}(\omega)\left(\varepsilon_{\alpha}+U / 2-\Sigma_{\alpha}\right),
$$

where $\bar{E}_{\alpha}=\varepsilon_{\alpha}+U\left(n_{\alpha}-1\right) / 2$. By using the semi-elliptic density of states (DOS) for noninteracting electrons, $\rho_{0}(\varepsilon)=\frac{2}{\pi W^{2}} \sqrt{W^{2}-\varepsilon^{2}}$, where $W$ is the half-width of the band to be set as the energy unit, we obtain a pair of equations for $G_{A}(\omega)$ and $G_{B}(\omega)$ :

$$
\begin{array}{r}
\frac{1}{16} G_{\bar{\alpha}}^{2}(\omega) G_{\alpha}(\omega)+\frac{1}{2}\left(\varepsilon_{\alpha}-\omega\right) G_{\bar{\alpha}}(\omega) G_{\alpha}(\omega)+\left[\left(\varepsilon_{\alpha}-\omega\right)^{2}-\frac{U^{2}}{4}\right] G_{\alpha}(\omega) \\
+\frac{1}{4} G_{\bar{\alpha}}(\omega)+\varepsilon_{\alpha}-\omega-\frac{U}{2}\left(n_{\alpha}-1\right)=0
\end{array}
$$

where the temperature-dependent occupation $n_{\alpha}=-2 / \pi \int_{-\infty}^{\infty} \frac{1}{e^{\frac{\omega}{T}}+1} \mathfrak{I} G_{\alpha}(\omega) d \omega$ (the chemical potential equals $U / 2$ due to the electron-hole symmetry in the half-filled system and we take this value as the origin of energy), $\alpha=A(B)$ and $\bar{\alpha}=B(A)$. The equations (5) must now be solved with $n_{A}+n_{B}=2$. From the self-consitent CPA solution of the IHM one can determine the local one-particle DOS $\rho_{\alpha}(\omega)=-\mathfrak{I} G_{\alpha}(\omega) / \pi$, the self-energy $\Sigma_{\alpha}(\omega)$ and the charge gap as functions of the model parameters $U, \Delta$ and temperature $T$.

\footnotetext{
${ }^{1}$ Delete "in this paper"
} 
The Kubo formula for the conductivity $\sigma$ involves the two-particle current-current response function. However in the local approximation of CPA or DMFT there is no vertex connection and $\sigma$ may be expressed in terms of the one-particle spectral function of the Green function

$$
A_{\sigma}(\varepsilon, \omega)=-\frac{1}{\pi} \mathfrak{J} \frac{1}{\varepsilon-\omega-\Sigma_{\sigma}(\varepsilon)} .
$$

To derive the conductivity of the half-filled IHM on a bipartite lattice, we follow the procedure described in Refs. [11,12], which gives

$$
\sigma=e^{2} \sum_{\sigma} \int d \varepsilon \rho_{0}(\varepsilon) \int d \omega\left(-\frac{d f(\omega)}{d \omega}\right) \mathrm{A}_{\mathrm{A} \sigma}(\varepsilon, \omega) \mathrm{A}_{\mathrm{B} \sigma}(\varepsilon, \omega)
$$

where $f(\omega)$ is the Fermi distribution function, $A_{\alpha, \sigma}(\varepsilon, \omega)$ is the one-particle spectral function of the Green function for the itinerant electron for $\alpha$-sublattice. Through this work we take the electron charge as the charge unit $(e=1)$.

\section{RESULTS AND DISCUSSION}

Before numerically solving equations (5) and calculating dc conductivity (7), let us consider the atomic limit $(t=0)$. In this case, when $U<2 \Delta$ the sites with lower energy $-\Delta$ are doubly occupied and those with higher energy $+\Delta$ are empty, therefore the IHM is a band insulator. In contrast, when $U>2 \Delta$ both types of sites are singly occupied and the IHM is a MI. At the single special value $U=2 \Delta$ the correlations close a charge gap and the system is in a metallic phase $[5,8]$. We turn now to present our numerical results for $t \neq 0$. Figure 1 shows the charge gap as a function of $U$ for three values of $\Delta$ at zero temperature. For $U<U_{c 1}(\Delta)$ we find the system is a BI with a gap of the order of $(2 \Delta-U)$. The gap is suppressed to zero at $U=U_{c 1}(\Delta)$ and remains zero within the metallic phase when $U_{c 1}(\Delta) \leq U \leq U_{c 2}(\Delta)$. For $U=U_{c 2}(\Delta)$ there is a second transition from the metal to a MI. That is when $t$ is nonzero the single metallic point is expanded to a finite range $\left[U_{c 1}, U_{c 2}\right]$. The corresponding conductivity between $U_{c 1}$ and $U_{c 2}$ for three values of $\Delta$ is plotted in Fig. 2. It is interesting to note that for intermediate and large $\Delta$ the largest conductivity occurs near the special value $U=2 \Delta$ as one might expect from the atomic limit case. Similar behavior of the conductivity in the half-filled IHM was also found in the DQMC study [5]. In addition, the largest conductivity $\sigma_{\max }(\Delta)$ decreases with increasing $\Delta$. In Fig. 3 we show the conductivity as a function of $U$ for $\Delta=0.75$ and for different values of $T$. Due to the Fermi distribution function, when $T$ increases the region of finite conductivity extends and its maximum decreases. However, the values of $U_{m} \approx 2 \Delta$ corresponding to the maximal conductivity are almost unchanged.

In a previous paper [10] we have found, in agreement with DQMC [5,6] and single-site DMFT [7-9], that MI and BI phases are sandwiched by a metallic one. This conclusion was obtained by the calculation of the DOS at the Fermi level. When the DOS shows a gap at the position of the Fermi level, the system is insulating. If the DOS is finite at the Fermi level the system is metallic. Here we show that this result is consistent with the calculated conductivity. In Fig. 4 we present the conductivity as a function of temperature $T$ for $\Delta=0.75$ and for different values of $U$. It can be seen that for $U=1.59$ the conductivity increases as $T$ is lowered, which implies that the system is metallic. In contrast, at $U=1.48<U_{c 1}$ and $U=1.84>U_{c 2}$ the conductivity goes to zero as $T$ is lowered, indicating insulating phases. 


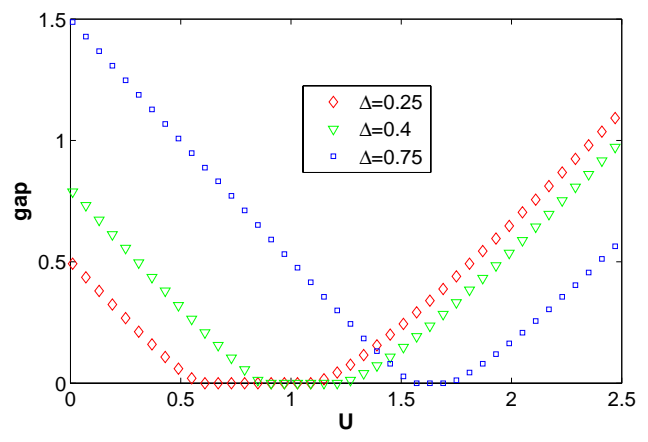

Fig. 1. Charge gap of IHM as a function of $U$ for $T=0$ and different values of $\Delta$.

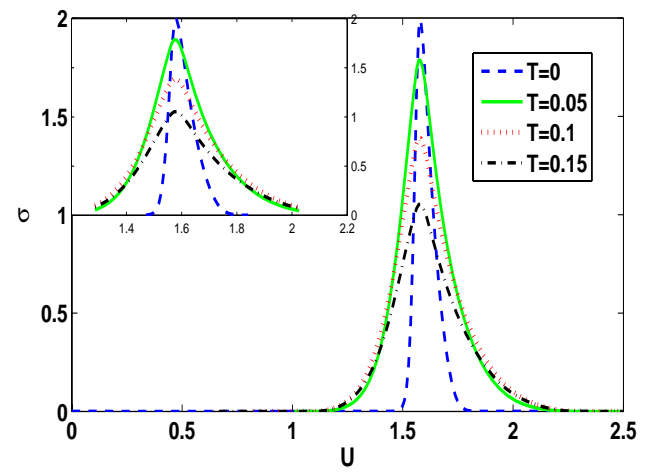

Fig. 3. Conductivity as a function of $U$ for $\Delta=$ 0.75 and different values of $T$.

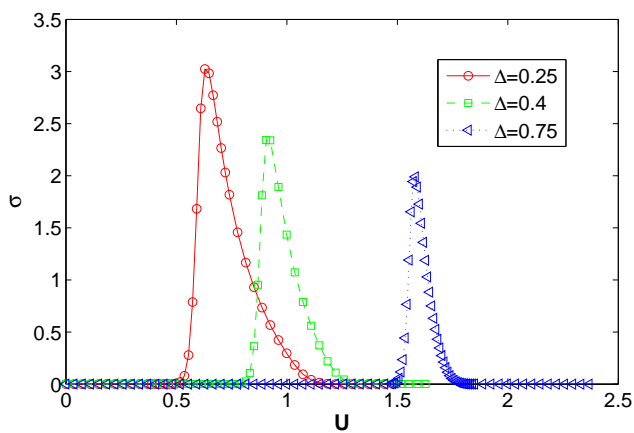

Fig. 2. Conductivity as a function of $U$ for $T=0$ and different values of $\Delta$.

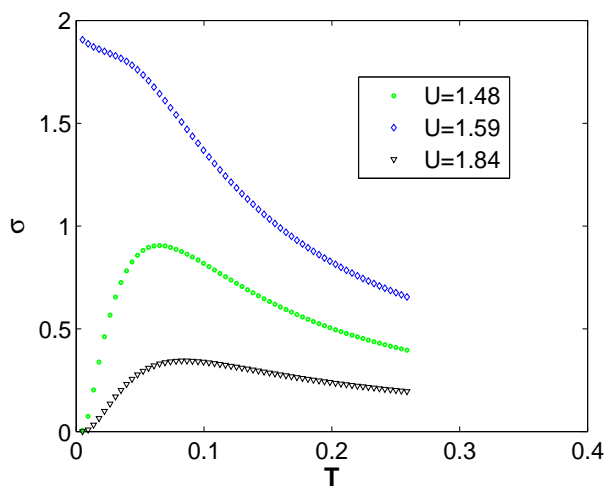

Fig. 4. Conductivity as a function of $T$ for $\Delta=$ 0.75 and different values of $U$.

\section{CONCLUSIONS}

We have studied the metal-insulator transition in the half-filled ionic Hubbard model within CPA. For a fixed and finite $\Delta$ two transitions from BI via metal to MI are found. The calculation of the temperature dependent conductivity demonstrated that for intermediate and large $\Delta$ the largest conductivity occurs near the special value $U=2 \Delta$ at all temperatures, for a fixed $\Delta$ the region of finite conductivity $\left[U_{c 1}, U_{c 2}\right]$ expands and its maximum decreases with increasing $T$. Our results are in good agreement with the ones obtained by the determinant quantum Monte Carlo simulation. The calculations presented here can be extended to the optical conductivity. This is left to future work.

\section{ACKNOWLEGMENTS}

One of us, NDT, would like to thank Dr. Pham Van Nham and Dr. Phung Duy Khuong for inspiring discussions at earlier stages of the present study. Financial support of the National Foundation for Science and Technology Development (NAFOSTED, project No 103.02-2011.05) is gratefully acknowledged. 


\section{REFERENCES}

[1] J. Hubbard, J.B. Torrance, Phys. Rev. Lett. 47 (1981) 1750.

[2] T. Egami, S. Ishihara, M. Tachiki, Science 261 (1993) 1307.

[3] K. Pozgajcic, C. Gros, Phys. Rev. B 68 (2003) 085106.

[4] D.A. Le, A.T. Hoang, Mod. Phys. Lett. B 26 (2012) 1150016.

[5] N. Paris et al., Phys. Rev. Lett. 98 (2007) 046403.

[6] K. Bouadim et al., Phys. Rev. B 76 (2007) 085112.

[7] K. Byczuk et al., Phys. Rev. B 79 (2009) 121103 (R).

[8] A. Garg, H.R. Krishnamurthy, M. Randeria, Phys. Rev. Lett. 97 (2006) 046403.

[9] L. Craco et al., Phys. Rev. B 78 (2008) 075121.

[10] A.T. Hoang, J. Phys.: Condens. Matter. 22 (2010) 095602.

[11] T. Pruschke, D.L. Cox, and M. Jarrell, Phys. Rev. B 47 (1993) 8553.

[12] V.N. Phan and M. T. Tran, Condmat/0310441 (2003). 